\title{
Article
}

\section{Minimum Time Problem Controlled by Affine Connection}

\author{
Constantin Udriste ${ }^{*, t, \ddagger(D)}$ and Ionel Tevy $\ddagger$ (D)
}

check for

updates

Citation: Udriste, C.; Tevy, I. Minimum Time Problem Controlled by Affine Connection. Symmetry 2021, 13, 1391. https://doi.org/10.3390/ sym 13081391

Academic Editor: Alexei Kanel-Belov

Received: 4 June 2021

Accepted: 9 July 2021

Published: 31 July 2021

Publisher's Note: MDPI stays neutral with regard to jurisdictional claims in published maps and institutional affiliations.

Copyright: (c) 2021 by the authors. Licensee MDPI, Basel, Switzerland. This article is an open access article distributed under the terms and conditions of the Creative Commons Attribution (CC BY) license (https:// creativecommons.org/licenses/by/ $4.0 /)$.
Department of Mathematics and Informatics, Faculty of Applied Sciences, University Politehnica of Bucharest, Splaiul Independentei 313, Sector 6,060042 Bucharest, Romania; tevy@mathem.pub.ro

* Correspondence: udriste@mathem.pub.ro; Tel.: +40-745-371-684

+ Second address: Academy of Romanian Scientists, Ilfov 3, Sector 5, 050044 Bucharest, Romania.

$\ddagger$ These authors contributed equally to this work.

\begin{abstract}
Geometrically, the affine connection is the main ingredient that underlies the covariant derivative, the parallel transport, the auto-parallel curves, the torsion tensor field, and the curvature tensor field on a finite-dimensional differentiable manifold. In this paper, we come up with a new idea of controllability and observability of states by using auto-parallel curves, and the minimum time problem controlled by the affine connection. The main contributions refer to the following: (i) autoparallel curves controlled by a connection, (ii) reachability and controllability on the tangent bundle of a manifold, (iii) examples of equiaffine connections, (iv) minimum time problem controlled by a connection, (v) connectivity by stochastic perturbations of auto-parallel curves, and (vi) computing the optimal time and the optimal striking time. The connections with bounded pull-backs result in bang-bang optimal controls. Some significative examples on bi-dimensional manifolds clarify the intention of our paper and suggest possible applications. At the end, an example of minimum striking time with simulation results is presented.
\end{abstract}

Keywords: affine connections; auto-parallel curves; minimum time optimal control; stochastic connectivity

MSC: 53B05; 49K15; 70Q05; 60H10

\section{Introduction}

Geometers [1,2] used the affine connection only in problems of differential geometry, especially in order to introduce and analyze the covariant derivative, the parallel transport, the auto-parallel curves, the torsion tensor field, and the curvature tensor field on finitedimensional differentiable manifolds. Since the difference $\nabla-{ }^{0} \nabla$ of two arbitrary affine connections is a tensor field in $\mathcal{T}_{2}^{1}(M)$, the space of all connections is an affine space over the vector space $\mathcal{T}_{2}^{1}(M)$. This idea can be represented by the formula $\nabla_{X} Y={ }^{0} \nabla_{X} Y+A(X, Y)$ (invariant form) or $\Gamma_{j k}^{i}(x)={ }^{0} \Gamma_{j k}^{i}(x)+A_{j k}^{i}(x), i, j, k=1, \ldots, n$ (using the components).

We emphasize that a connection controls the optimal time in which a given state is reached through an auto-parallel curve starting from a fixed point and by following the technique of Evans in the Lecture Notes [3]. Section 2 discusses the controlled ODEs of auto-parallel curves. Section 3 analyzes the reachable set and controllability on the tangent bundle of a manifold. Section 4 underlines some equiaffine connections obtained by adapted dynamical systems. This Section closes with examples of bi-dimensional Riemannian manifolds possessing a constant determinant of the metric matrix. Section 5 provides an answer for the minimum time problem controlled by a connection. Section 6 refers to important examples (bounded connection, constant connection, and polynomial connection) on bi-dimensional manifolds. Section 7 develops another variant of minimum time problem. Section 8 studies the stochastic connectivity based on a stochastic perturbation of Pfaff ODEs describing the auto-parallels. Here, the following issues are emphasized: Pfaff form of auto-parallel ODEs, stochastic perturbations, the simplified stochastic minimum principle, and striking time. 
Bang-bang control, where the input switches between upper and lower bounds, is the optimal strategy for solving a wide variety of control problems where the control of a dynamical system has lower and upper bounds and the problem is non-singular and of degree one in the input. Bang-bang type controls arise in many applications, for example, applied physics [4,5], game theory [6], chemistry [7], biology [8], socio-economic systems [9], minimum-time problems [10], etc. The paper [11] refers to optimal control for mechanical systems on Riemannian manifolds and the paper [12] is dedicated to the local, linear, global, and exact controllability and observability.

Although there is an overlap of words, our point of view is completely different from the one in the papers [13] (affine connection control systems) and [14] (the category of affine connection control systems): (1) In our paper, the affine connection is a control; (2) in the papers $[13,14]$, the affine connection defines the covariant derivative and the control enters linearly in the second member of ODEs.

Bang-bang connection control implies constant connections. For their meaning, we recall that there exist constant connections that are solutions for curvature-flatness PDEs $[15,16]$.

\section{Controlled ODEs of Auto-Parallel Curves}

Let $(M, \nabla)$ be an $n$-dimensional affine manifold and $\Gamma_{j k}^{i}(x) \in \mathcal{F}(M), i, j, k=\overline{1, n}$, be the components of the connection $\nabla$. To find the auto-parallel curves we use the pull-back $\gamma(t)=\Gamma(x(t))$ and we solve the ODEs system of the auto-parallel curves (see the Einstein summation convention) using the following equation

$$
\ddot{x}^{i}(t)+\Gamma_{j k}^{i}(x(t)) \dot{x}^{j}(t) \dot{x}^{k}(t)=0 .
$$

Without loss of generality, we can only consider symmetric connection because the antisymmetric part is destroyed in the expression $\Gamma_{j k}^{i}(x(t)) \dot{x}^{j}(t) \dot{x}^{k}(t)$.

If we force the pull-back $\gamma(t)$, we cannot recover the connection $\nabla$ of components $\Gamma_{j k}^{i}(x)$, except in the case of a null connection.

\subsection{Auto-Parallelly Complete Manifold}

The connection $\nabla$ of components $\Gamma_{i j}^{h}(x)$ produces auto-parallel curves $x(t)$ via second order ODEs system (1) and associated bilocal problems (particularly Sturm-Liouville problems).

Definition 1. An affine manifold $(M, \nabla)$ is called auto-parallelly complete if any auto-parallel $x(t)$ starting at $p \in M$ is defined for all values of the parameter $t \in \mathbb{R}$.

Theorem 1. [1,17] Let $M$ be a (Hausdorff, connected, and smooth) compact n-manifold endowed with an affine connection $\nabla$ and let $p \in M$. If the holonomy group $\operatorname{Hol}_{p}(\nabla)$ (regarded as a subgroup of the group $G l\left(T_{p} M\right)$ of all the linear automorphisms of the tangent space $\left.T_{p} M\right)$ has compact closure, then $(M, \nabla)$ is auto-parallelly complete.

Classically, the auto-parallel curves are used for defining the convexity of subsets $D$ in $M$ and convexity of functions $f: D \subset M \rightarrow \mathbb{R}$. Here we develop new ideas: (i) controllability and observability of states through auto-parallel curves, (ii) solving the minimum time problem controlled by the affine connection, and (iii) establishing stochastic connectivity via stochastic perturbations of auto-parallel Pfaff ODEs.

\subsection{Prolongation to Tangent Bundle}

We transform the second order ODEs system (1) on the manifold $M$ into a first order ODEs system on the tangent bundle $T M$. In this manner, we obtain a natural lift of auto-parallel curves. 
In other words, the nonlinear control systems that will be considered in this Section are first order systems of the following form:

$$
\dot{x}^{i}(t)=y^{i}(t), \dot{y}^{i}(t)=-\Gamma_{j k}^{i}(x(t)) y^{j}(t) y^{k}(t), i, j, k=1, \ldots, n,
$$

where $(x, y) \in T M$ and $\gamma_{j k}^{i}(t)=\Gamma_{j k}^{i}(x(t))$ are the feed-back control on $M$. The set of all feed-back controls $\{\gamma\}$ is not a vector space. By introducing the matrix $A(x(t), y(t))=$ $\left(\Gamma_{j k}^{i}(x(t)) y^{j}(t)\right)$, we can write an implicit form

$$
\dot{x}(t)=y(t), \dot{y}(t)=-A(x(t), y(t)) y(t),
$$

where $A(x(t), 0)=O$ or a matrix form

$$
\left(\begin{array}{l}
\dot{x} \\
\dot{y}
\end{array}\right)=\left(\begin{array}{cc}
O & I \\
O & -A(x, y)
\end{array}\right)\left(\begin{array}{l}
x \\
y
\end{array}\right)
$$

Open problem: Is the non-isolated equilibrium point $\left(x_{0}, 0\right)$ stable or unstable?

Theorem 2. Let us consider the Riemannian manifold $(M, g)$ and the associated Sasaki tangent bundle ( $T M, G=g \oplus g)$. Then, the non-isolated equilibrium point $\left(x_{0}, 0\right) \in T M$ is stable.

Proof. The function $F: T M \rightarrow \mathbb{R}, F(x, y)=\frac{1}{2} g_{i j}(x) y^{i} y^{j}$ is a first integral. To verify this statement, we compute the following

$$
\frac{d}{d t} F(x(t), y(t))=\frac{1}{2} \frac{\partial g_{i j}}{\partial x^{k}} y^{i} y^{j} y^{k}-g_{i m} y^{m} \Gamma_{j k}^{i} y^{j} y^{k}
$$

Since the connection can be written

$$
\Gamma_{j k}^{i}=\frac{1}{2} g^{i l}\left(\delta_{l}^{r} \delta_{j}^{s} \delta_{k}^{t}+\delta_{l}^{r} \delta_{k}^{s} \delta_{j}^{t}-\delta_{l}^{t} \delta_{j}^{r} \delta_{k}^{s}\right) \frac{\partial g_{r s}}{\partial x^{t}},
$$

it follows $\frac{d}{d t} F(x(t), y(t))=0$ and hence $F$ is a Lyapunov function.

\section{Reachable Set and Controllability by Lift of Auto-Parallel Curves}

Part of our inspiration comes from the classic works on reachable set and controllability $[18,19]$. The new idea is to introduce and study these notions for natural lift of auto-parallel curves.

Let us consider an auto-parallelly complete affine manifold $(M, \nabla)$ and the nonlinear control system (2) on the tangent bundle TM.

Definition 2 (Reachable set). We fix the point $\left(x_{0}, y_{0}\right) \in T M$ and let $V \subset T M$ be a neighborhood of the point $\left(x_{0}, y_{0}\right)$.

(a) The $V$-reachable set $R_{V}\left(\left(x_{0}, y_{0}\right), T\right)$ is the set of all points that can be reached from $\left(x_{0}, y_{0}\right)$ at time $T>0$ following the lift of auto-parallel curves, which remains in $V$ for $0 \leq t \leq T$, i.e.,

$$
\begin{gathered}
R_{V}\left(\left(x_{0}, y_{0}\right), T\right)=\{(x, y) \in T M \mid \exists \nabla \text { such that } \\
\left.(x, y)\left(t,\left(x_{0}, y_{0}\right), \nabla\right) \in V \text { and }(x, y)\left(T,\left(x_{0}, y_{0}\right), \nabla\right)=(x, y)\right\} .
\end{gathered}
$$

(b) The $V$-reachable set at time $\tau \leq T$, which is denoted by $R_{V \tau}\left(x_{0}, y_{0}\right)$, is defined by

$$
R_{V \tau}\left(x_{0}, y_{0}\right)=\cup_{t \leq \tau} R_{V}\left(\left(x_{0}, y_{0}\right), \tau\right) .
$$

(c) The reachable set $R\left(x_{0}, y_{0}\right)$ from $\left(x_{0}, y_{0}\right)$ is

$$
R\left(x_{0}, y_{0}\right)=\cup_{T>0} R_{M}\left(\left(x_{0}, y_{0}\right), T\right) .
$$


Definition 3 (Controllability). (a) The nonlinear control system (2) is called controllable if for every point $\left(x_{0}, y_{0}\right) \in T M$ we have that $R\left(x_{0}, y_{0}\right)=T M$.

(b) Let $\left(x_{0}, y_{0}\right) \in T M$ be given. Then, the nonlinear control system (2) is called locally controllable from the point $\left(x_{0}, y_{0}\right)$ if, for every neighborhood $V$ of $\left(x_{0}, y_{0}\right)$ and every $T>0$, the set $R_{V T}\left(x_{0}, y_{0}\right)$ contains a neighborhood of $\left(x_{0}, y_{0}\right)$.

Controllability does not imply reachability. Completeness assures us that reachability always implies controllability.

\section{Equiaffine Connections}

An equiaffine connection is an affine connection on an $n$-dimensional manifold $M$ for which there is a non-zero $n$-form $\omega$ on $M$ that is covariantly constant with respect to the given connection (volume form). Particular equiaffine connections are found in this section exploiting special dynamical systems.

\section{Two Geometric Corollaries to Adapted Theorems}

Theorem 3. Let us consider the matrix dynamical system $\dot{Y}=A(Y) Y$, where the unknown $Y$ can be a vector or a matrix (not necessarily square). If the matrix $A(Y)$ is antisymmetric, then the square form $Q(Y)=Y^{T} Y$ is invariant (first integral). Particularly, if the initial value $Y(0)=Y_{0}$ consists in orthonormal columns, i.e., $Y_{0}^{T} Y_{0}=I$, then the columns of the solution $Y(t)$ are orthonormal vectors.

Proof. We calculate the derivative of the function $Q(Y)$ along the direction $H$. We find $Q^{\prime}(Y) H=Y^{T} H+H^{T} Y$. We replace $H=A(Y) Y$. Consequently, $Q^{\prime}(Y) A(Y) Y=Y^{T} A(Y) Y$ $+(A(Y) Y)^{T} Y=0$.

Let us find some geometric consequences. Given the auto-parallel $x(t)$ and the pull-back $\Gamma_{j k}^{i}(x(t))$, we consider the following differential system

$$
\dot{y}^{i}(t)=-\Gamma_{j k}^{i}(x(t)) y^{j}(t) y^{k}(t) .
$$

Denoting $Y^{m n}=y^{m} y^{n}$, we build a new differential system $\dot{\Upsilon}^{m n}=-\left(\Gamma_{j k}^{m} y^{n}+\Gamma_{j k}^{n} y^{m}\right) Y^{j k}$, with the matrix $A^{m n}{ }_{j k}(\Gamma, y)=-\left(\Gamma_{j k}^{m} y^{n}+\Gamma_{j k}^{n} y^{m}\right)$.

Corollary 1. If the matrix $A=\left(A^{m n}{ }_{j k}\right)$ is antisymmetric, then (i) the quadratic form $Q(Y)=$ $Y^{T} Y$ is invariant (first integral); (ii) the trace $\Gamma_{i j}^{i}$ of the connection 1-form vanishes and hence the Ricci tensor field produced by the connection $\nabla$ is symmetric; (iii) the Riemannian volume element of the manifold $\left(M, g_{i j}\right)$ is constant.

Proof. Explicitly, using the following matrix language: $y$ line matrix; $Y=y^{T} y$ and

$$
Q(Y)=Y^{T} Y=\left(y^{T} y\right)^{T}\left(y^{T} y\right)=\left(y^{T} y\right)^{2}=Y^{2} .
$$

The antisymmetry of the matrix $A=\left(A^{m n}{ }_{j k}\right)$ means the following:

$$
\Gamma_{j k}^{m}(x) y^{n}+\Gamma_{j k}^{n}(x) y^{m}=-\Gamma_{m n}^{j}(x) y^{k}-\Gamma_{m n}^{k}(x) y^{j}, \forall(x, y) \in T M,
$$

In other words,

$$
\Gamma_{j k}^{m}(x) \delta_{l}^{n}+\Gamma_{j k}^{n}(x) \delta_{l}^{m}+\Gamma_{m n}^{j}(x) \delta_{l}^{k}+\Gamma_{m n}^{k}(x) \delta_{l}^{j}=0 .
$$

By contraction $(m, n) \rightarrow(j, k)$, it follows that the trace $\Gamma_{i j}^{i}$ of the connection 1-form vanishes. Consequently the connection is equiaffine and the Ricci tensor field $R_{i j}=$ $\partial_{r} \Gamma_{i j}^{r}+\Gamma_{r i}^{l} \Gamma_{l j}^{r}$ is symmetric. 
Let $\left(M, g=\left(g_{i j}\right)\right)$ be a Riemannian manifold. The Riemannian metric $\left(g_{i j}\right)$ determines a symmetric connection (Levi-Civita connection) of components

$$
\Gamma_{j k}^{i}=\frac{1}{2} g^{i l}\left(\delta_{l}^{r} \delta_{j}^{s} \delta_{k}^{t}+\delta_{l}^{r} \delta_{k}^{s} \delta_{j}^{t}-\delta_{l}^{t} \delta_{j}^{r} \delta_{k}^{s}\right) \frac{\partial g_{r s}}{\partial x^{t}} .
$$

Taking the trace $i \rightarrow j$, we find

$$
\Gamma_{i k}^{i}=\frac{1}{2} g^{r s} \frac{\partial g_{r s}}{\partial x^{k}}=\frac{1}{\sqrt{G}} \frac{\partial}{\partial x^{k}} \sqrt{G}=0, G=\operatorname{det}\left(g_{i j}\right) .
$$

If $\Gamma_{i k}^{i}=0$, then the determinant $G$ of the metric $g_{i j}$ is constant.

Theorem 4. Let us consider the matrix dynamical system $\dot{Y}=A(Y) Y, Y(0)=Y_{0}$, where the matrix $A(Y)$ and the unknown $Y$ are square matrices. If the matrix $A(Y)$ has zero trace, then the determinant $F(Y)=\operatorname{det} Y$ is a first integral (invariant function).

Proof. By calculation we have the following

$$
\operatorname{det}(Y+(\exp A) Y)=\operatorname{det}(I+\exp A) \operatorname{det} Y=\left(1+\exp \operatorname{tr} A+\mathcal{O}\left(\exp ^{2}\right)\right) \operatorname{det} Y .
$$

It follows $F^{\prime}(Y) A Y=\operatorname{tr} A \operatorname{det} Y$. Then, $F^{\prime}(Y) A Y=0$.

Now we are interested in finding some geometric consequences.

Corollary 2. If the matrix $A^{m n}{ }_{j k}(\nabla, y)$ has zero trace, then (i) the trace $\Gamma_{i j}^{i}$ of the connection 1-form vanishes; (ii) the Ricci tensor field produced by the connection $\nabla$ is symmetric; (iii) the Riemannian volume element of the manifold $\left(M, g_{i j}\right)$ is constant.

Proof. We recall $A^{m n}{ }_{j k}(\nabla, y)=-\left(\Gamma_{j k}^{m} y^{n}+\Gamma_{j k}^{n} y^{m}\right)$. The trace condition $A_{j k}^{j k}=0, \forall y$ implies $\Gamma_{i j}^{i}=0$.

Consequently, for the Levi-Civita connection with such property attached to the metric $g_{i j}$, the determinant $G$ of the metric $g_{i j}$ is constant.

Example 1. (i) Let us consider the Riemannian manifold $\left(\mathbb{R}^{2}, g_{i j}\right)$, where $g_{11}=f(x, y) a_{11}$, $g_{12}=a_{12}, g_{22}=\frac{1}{f(x, y)} a_{22}, f(x, y)>0,\left(a_{i j}\right)=$ constant positive definite matrix. Then $G=$ $\operatorname{det}\left(g_{i j}\right)=a_{11} a_{22}-a_{12}^{2}$ is constant.

(ii) The Riemannian metric $g_{11}(x, y)=\cosh \left(x^{2}+y^{2}\right), g_{12}(x, y)=\sinh \left(x^{2}+y^{2}\right), g_{22}(x, y)$ $=\cosh \left(x^{2}+y^{2}\right),(x, y) \in \mathbb{R}^{2}$ has the determinant $G=1$.

(iii) Let's take a surface $z=f(x, y)$ in $\mathbb{R}^{3}$. The constant area element condition returns to an Eikonal PDE $\left(\frac{\partial f}{\partial x}\right)^{2}+\left(\frac{\partial f}{\partial y}\right)^{2}=$ ct. We replace this PDE by the PDEs system $\frac{\partial f}{\partial x}=$ $\sin g(x, y), \frac{\partial f}{\partial y}=\cos g(x, y)$. The condition of complete integrability $\frac{\partial g}{\partial x} \sin g(x, y)+\frac{\partial g}{\partial y} \cos g(x, y)$ $=0$ is identically checked only for $g(x, y)=c t=\alpha$. The surface is a plane $z=x \sin \alpha+y \cos \alpha$, with the Euclidean metric.

Generally, the Eikonal PDE $\|\nabla u(x)\|^{2}=v(x), x=\left(x^{1}, \ldots, x^{n}\right) \in \mathbb{R}^{n}$ is related to geometric optics (rays) and admits viscosity solutions.

\section{Minimum Time Problem Controlled by Connection}

Let us consider a compact affine manifold $(M, \nabla)$ of dimension $n$, where $\nabla$ is a symmetric connection with the components $\Gamma_{j k}^{i}(x), i, j, k=1, \ldots, n$. The number of independent components of the connection is $\frac{n^{2}(n+1)}{2}$. The control constraint $\left|\Gamma_{j k}^{i}(x(t))\right| \leq 1, t \in\left[t_{0}, T\right]$ for each triple $(i, j, k)$ means a hyper-cube with $2^{n^{2}(n+1)}$ vertices. 
Objective: Find the feedback optimal control $\gamma_{j k}^{i}(t)=\Gamma_{j k}^{i}(x(t))$ to transfer the system (2) from a given initial point $\left(x\left(t_{0}\right), y\left(t_{0}\right)\right) \in T M$ to a (possibly moving) target set $S[(x(T), y(T)), T]$ in the shortest possible time, via the following functional

$$
J=\int_{t_{0}}^{T} d t=T-t_{0}
$$

State Constraints: Controlled nonlinear first order ODEs system

$$
\dot{x}^{i}(t)=y^{i}(t), \dot{y}^{i}(t)=-\Gamma_{j k}^{i}(x(t)) y^{j}(t) y^{k}(t), i, j, k=1, \ldots, n,
$$

with unknown $(x(t), y(t))$. Boundary conditions (Sturm-Liouville problems) are

$$
\left(x\left(t_{0}\right), y\left(t_{0}\right)\right)=\left(x_{0}, y_{0}\right),\left(x\left(T^{*}\right), y\left(T^{*}\right)\right) \in S\left[\left(x\left(T^{*}\right), y\left(T^{*}\right)\right), T^{*}\right] .
$$

The control constraints are the following: $t \in\left[t_{0}, T\right],|\gamma(t)| \leq 1$.

Reachability: What states can be reached from the initial condition $\left(x_{0}, y_{0}\right)$ in a finite time?

Problem data: $\left.\Gamma_{j k}^{i}(x(t))\right|_{\left[t_{0}, t\right]^{-}}$admissible control in the time interval $\left[t_{0}, t\right] ; A_{t^{-}}$those states that can be reached at time $t$ from the point $\left(x\left(t_{0}\right), y\left(t_{0}\right)\right)$ by an admissible control; for each $t$, the set $A_{t}$ is closed and bounded and $A_{t}$ grows with time, i.e., $t_{0}<t_{1}<t_{2} \Rightarrow$ $x\left(t_{0}\right) \in A_{t_{1}} \subset A_{t_{2}} ; S_{t^{-}}$the set of target states at time $t ; T^{*}$ - minimum time $=$ time of first intersection of the sets $S_{t}$ and $A_{t}$.

Defining minimum time: (i) no solution exists if $A_{t} \cap S_{t}=\varnothing, \forall t \geq t_{0}$; (ii) if $A_{t_{i}} \cap S_{t_{i}} \neq$ $\varnothing$ and $A_{t} \cap S_{t}=\varnothing, \forall t<t_{i}$, then $t_{i}=T^{*}$ is the minimum time solution = time of first intersection of $A_{t}$ and $S_{t}$.

Solution procedure: (i) Pontryagin's Minimum Principle provides the necessary conditions for $\Gamma_{j k}^{* i}(x(t))$; (ii) same controlled ODEs system, control constraints, and performance index as previously shown; (iii) the target set is as follows

$$
g[(x, y), T]=0 \Rightarrow\left(x\left(T^{*}\right), y\left(T^{*}\right)\right) \in S((x, y), T) .
$$

The solution procedure follows that for the general continuous time optimal control problem, the following is performed: define the Hamiltonian; find the necessary conditions; place the control law in feed-back form.

The associated Hamiltonian is

$$
H(x(t), y(t), \lambda(t), \mu(t), \Gamma(x(t)), t)=1+\lambda_{i}(t) y^{i}(t)-\mu_{i}(t) \Gamma_{j k}^{i}(x(t)) y^{j}(t) y^{k}(t) .
$$

The Hamiltonian is of degree one in the feed-back control $\gamma_{j k}^{i}(t)=\Gamma_{j k}^{i}(x(t))$ and the set of all feed-back controls $\{\gamma\}$ is not a vector space. The switch coefficients are the following (decomposition as a tensor product)

$$
\sigma_{i}^{j k}(t)=-\mu_{i}(t) y^{j}(t) y^{k}(t)
$$

Since the problem is characterized by degree one in control and the control set is closed, we can expect boundary solutions to occur.

Necessary conditions: Initial dynamics ODEs

$$
\dot{x}^{i}(t)=\frac{\partial H}{\partial \lambda_{i}}=y^{i}(t), \dot{y}^{i}(t)=\frac{\partial H}{\partial \mu_{i}}=-\Gamma_{j k}^{i}(x(t)) y^{j}(t) y^{k}(t) ;
$$

Bilocal conditions $\left(x\left(t_{0}\right), y\left(t_{0}\right)\right)=\left(x_{0}, y_{0}\right), g\left(\left(x\left(T^{*}\right), y\left(T^{*}\right)\right), T^{*}\right)=0$. 
Adjoint dynamics ODEs

$$
\begin{gathered}
\dot{\lambda}_{i}(t)=-\frac{\partial H}{\partial x^{i}}=\mu_{i}(t) \frac{\partial \Gamma_{j k}^{i}}{\partial x^{l}}(x(t)) y^{j}(t) y^{k}(t) y^{l}(t) \\
\dot{\mu}_{i}(t)=-\frac{\partial H}{\partial y^{i}}=-\lambda_{i}+2 \mu_{l} \Gamma_{i k}^{l}(x(t)) y^{k}(t) ;
\end{gathered}
$$

Transversality conditions $\lambda_{i}\left(T^{*}\right)=0, \mu_{i}\left(T^{*}\right)=0$.

Control Constraints: $\left|\gamma_{j k}^{i}(t)\right|=\left|\Gamma_{j k}^{i}(x(t))\right| \leq 1, t \in\left[t_{0}, T\right], i, j, k=1, \ldots, n$.

Finding the Optimal Control (Bang-Bang control): the minimum condition:

$$
\begin{gathered}
H\left(x^{*}(t), y^{*}(t), \lambda^{*}(t), \mu^{*}(t), \Gamma^{*}\left(x^{*}(t)\right), t\right) \\
\leq H\left(x^{*}(t), y^{*}(t), \lambda^{*}(t), \mu^{*}(t), \Gamma(x(t)), t\right), \quad \forall \text { admissible } \Gamma(x(t)),
\end{gathered}
$$

is equivalent to the following maximum condition

$$
\mu_{i}^{*}(t) \Gamma_{j k}^{* i}\left(x^{*}(t)\right) y^{* j}(t) y^{* k}(t) \geq \mu_{i}^{*}(t) \Gamma_{j k}^{i}(x(t)) y^{* j}(t) y^{* k}(t), \quad \forall \text { admissible } \Gamma(x(t)) .
$$

On the other hand,

$$
\max _{\left|\Gamma_{j k}^{i}(x(t))\right| \leq 1} \mu_{i}^{*}(t) \Gamma_{j k}^{i}(x(t)) y^{* j}(t) y^{* k}(t)=\left|\mu_{i}^{*}(t) y^{* j}(t) y^{* k}(t)\right|
$$

and the optimal connections are those with particular constant feed-back. More precisely, for each triplet $(i, j, k)$, we have

$$
\gamma_{j k}^{* i}(t)=\Gamma_{j k}^{* i}\left(x^{*}(t)\right)=\operatorname{sign}\left(-\mu_{i}^{*}(t) y^{* j}(t) y^{* k}(t)\right) .
$$

Connections with particular constant coefficients are examples. Connections with constant feed-back (constant on auto-parallel curves) do not mean constant connections.

The control is always at its extremum value. Bang-bang solutions arise since the Hamiltonian is of degree one in the control variable:

$$
\gamma_{j k}^{* i}(t)=\operatorname{sign} \sigma_{i}^{j k}(t)=\left\{\begin{array}{cccc}
1 & \text { for } & \sigma_{i j k}^{j k}(t)<0: & \text { bang-bang control } \\
\gamma_{s g} & \text { for } & \sigma_{i j k}^{j k}(t)=0: & \text { singular control } \\
-1 & \text { for } & \sigma_{i}^{j k}(t)>0: & \text { bang-bang control. }
\end{array}\right.
$$

"Normal" minimum time problem: Let 0 denote the null section of the tangent bundle. Denote by $\widetilde{T M}=T M \backslash\{0\}$. Suppose, on $\widetilde{T M}$, the switch coefficients

$$
\sigma_{i}^{j k}(t)=-\mu_{i}^{*}(t) y^{* j}(t) y^{* k}(t)
$$

are 0 along the lift of an auto-parallel curve $(x(t), y(t))$ and only at isolated points $\tau$ in time, i.e., $\mu_{i}^{*}(\tau)=0, \forall i=1, \ldots, n$.

"Singular" minimum time problem: On $\widetilde{T M}$, some of the switch coefficients $\sigma_{i}^{j k}(t)$ are 0 over a time interval with non-zero length. Then, all components of the optimal connection are null except those that are determined by sign. The choice of optimal $\gamma^{*}$ must be obtained from other information than "max w.r.t. $\gamma$ ".

\section{Bidimensional Examples}

Example 2. To clarify some notions, we start with examples on the bi-dimensional manifold $\left(\mathbb{R}^{2}, \Gamma_{j k}^{i}(x)\right)$, i.e., $n=2$ and $x=\left(x^{1}, x^{2}\right)$. 


\subsection{Bounded Connection}

Let us take an example of bounded connection (no relation with optimal control)

$$
\Gamma_{11}^{1}(x)=\Gamma_{12}^{1}(x)=\Gamma_{22}^{1}(x)=\sin \left(x^{1}\right) ; \Gamma_{11}^{2}(x)=\Gamma_{12}^{2}(x)=\Gamma_{22}^{2}(x)=\cos \left(x^{2}\right) .
$$

Then, globally, $\left|\Gamma_{j k}^{i}(x)\right| \leq 1$. The auto-parallel ODEs system is

$$
\ddot{x}^{1}+\sin \left(x^{1}\right) \cdot\left(\dot{x}^{1}+\dot{x}^{2}\right)^{2}=0, \ddot{x}^{2}+\cos \left(x^{2}\right) \cdot\left(\dot{x}^{1}+\dot{x}^{2}\right)^{2}=0 .
$$

We find

$$
\frac{\ddot{x}^{1}(t)}{\sin \left(x^{1}(t)\right)}=\frac{\ddot{x}^{2}(t)}{\cos \left(x^{2}(t)\right)} .
$$

Consequently, we obtain the following (pendulum ODEs):

$$
\frac{\ddot{x}^{1}(t)}{\sin \left(x^{1}(t)\right)}=-k^{2}, \frac{\ddot{x}^{2}(t)}{\cos \left(x^{2}(t)\right)}=-k^{2},
$$

where $k=\dot{x}^{1}+\dot{x}^{2}$ or $x^{1}(t)+x^{2}(t)=k t+c$.

\subsection{Constant Connection}

Suppose a constant bounded connection of components

$$
\begin{aligned}
& \Gamma_{11}^{1}(x)=\Gamma_{12}^{1}(x)=\Gamma_{22}^{1}(x)=\sin (\alpha), \\
& \Gamma_{11}^{2}(x)=\Gamma_{12}^{2}(x)=\Gamma_{22}^{2}(x)=\cos (\alpha) .
\end{aligned}
$$

Then, $\left|\Gamma_{j k}^{i}(x)\right| \leq 1$. In this case, the auto-parallels are solutions of the non-autonomous ODEs system

$$
\ddot{x}^{1}+\sin (\alpha) \cdot\left(\dot{x}^{1}+\dot{x}^{2}\right)^{2}=0, \ddot{x}^{2}+\cos (\alpha) \cdot\left(\dot{x}^{1}+\dot{x}^{2}\right)^{2}=0 .
$$

We get

$$
\begin{gathered}
x^{1}(t) \cos (\alpha)=x^{2}(t) \sin (\alpha)+c_{1} t+c_{2} \\
\ddot{x}^{2}(t)+\dot{x}^{2}(t)(\cos (\alpha)+\sin (\alpha))+c_{1}
\end{gathered}
$$

and the auto-parallels that result are described as follows

$$
\begin{gathered}
x^{1}(t)=x^{2}(t) \tan (\alpha)+\frac{c_{1} t+c_{2}}{\cos (\alpha)} \\
x^{2}(t)=\frac{c_{3} e^{-(\sin (\alpha)+\cos (\alpha)) t}}{\sin (\alpha)+\cos (\alpha)}+\frac{c_{1} t}{\sin (\alpha)+\cos (\alpha)}+c_{4} .
\end{gathered}
$$

\subsection{Polynomial Connection}

The inequalities $\left|\Gamma_{j k}^{i}(x)\right| \leq 1$ show that the values $\Gamma_{j k}^{i}(x)$ are in the hyper-cube $[-1,1]^{6}$. If we accept the following connection

$$
\Gamma_{11}^{1}(x)=\Gamma_{12}^{1}(x)=\Gamma_{22}^{1}(x)=x^{1} ; \Gamma_{11}^{2}(x)=\Gamma_{12}^{2}(x)=\Gamma_{22}^{2}(x)=x^{2},
$$

then, the auto-parallel ODEs system is

$$
\ddot{x}^{1}+x^{1} \cdot\left(\dot{x}^{1}+\dot{x}^{2}\right)^{2}=0, \ddot{x}^{2}+x^{2} \cdot\left(\dot{x}^{1}+\dot{x}^{2}\right)^{2}=0 .
$$

The solution

$$
x^{1}(t)=C_{2} t+C_{3}, x^{2}(t)=-x^{1}(t)+C_{1}
$$


is obtained. It follows

$$
\frac{\ddot{x}^{1}(t)}{x^{1}(t)}=\frac{\ddot{x}^{2}(t)}{x^{2}(t)}=-k^{2} \text { (pendulum), } \dot{x}^{1}(t)+\dot{x}^{2}(t)=k .
$$

Generally we have

$$
\frac{\ddot{x}^{1}+\ddot{x}^{2}}{\left(\dot{x}^{1}+\dot{x}^{2}\right)^{2}}=-\left(x^{1}+x^{2}\right) .
$$

Denoting $z=x^{1}+x^{2}$, this ODE becomes

$$
\frac{\ddot{z}}{\dot{z}}=-z \dot{z},
$$

whence $\ln \dot{z}=-\frac{z^{2}}{2}+k_{1}$ or $\dot{z}=k \exp \left(-\frac{z^{2}}{2}\right)$ or

$$
z(t)=-I \operatorname{RootO} f\left(I k \sqrt{2} C_{1}+I k \sqrt{2} t-\operatorname{erf}(z(t)) \sqrt{\pi}\right) \sqrt{2} .
$$

Example 3 (Bang-Bang). The feedback bounded control is $\gamma(t)=\Gamma(x(t))$. We can think alternately, either in the control $\Gamma(x)$ or in its feedback $\gamma(t)$. The control hyper-cube $|\gamma(t)| \leq 1$ has $2^{12}$ vertices.

First vertex: Suppose $\Gamma_{11}^{1}=\ldots=\Gamma_{22}^{2}=1$ on whole manifold. Then the auto-parallel $O D E$ system is

$$
\ddot{x}^{1}+\left(\dot{x}^{1}+\dot{x}^{2}\right)^{2}=0, \ddot{x}^{2}+\left(\dot{x}^{1}+\dot{x}^{2}\right)^{2}=0
$$

and implies $x^{1}(t)=x^{2}(t)+a t+b, \dot{x}^{1}+\dot{x}^{2}=\frac{1}{2} \ln k(2 t+m)$, whence the general solution is

$$
x^{1}(t)=\frac{1}{2}\left(\frac{1}{2} \ln k(2 t+m)+a t+b\right), x^{2}(t)=\frac{1}{2}\left(\frac{1}{2} \ln k(2 t+m)-a t-b\right) .
$$

We select an auto-parallel starting from the point $\left(x\left(t_{0}\right), y\left(t_{0}\right)\right)=\left(x_{0}, y_{0}\right)$ and ending at origin, i.e., $x^{1}(T)=0, x^{2}(T)=0$. Then, the optimal time is $T=-\frac{b}{a}, a b<0$ if $k\left(m-\frac{2 b}{a}\right)=1$. If not, we must have a switch and we must pass to a new vertex of control hyper-cube (new connection).

Determine the dual functions $\lambda_{i}(t)$, and $\mu_{i}(t)$ in case of the first vertex: Replacing in the general formulas, we find $\lambda_{i}(t)=0, i=1,2$ and $\dot{\mu}_{i}(t)=2\left(\mu_{1}(t)+\mu_{2}(t)\right)\left(y^{1}(t)+y^{2}(t)\right), i=$ 1, 2. It follows $\mu_{1}(t)+c=\mu_{2}(t)$ and $2 \mu_{1}(t)+c=4 \exp \left(\int_{t_{0}}^{t}\left(y^{1}(s)+y^{2}(s)\right) d s\right)$, where $y^{i}(s)=$ $\dot{x}^{i}(s), i=1,2$. The transversality condition $\mu_{i}\left(T^{*}\right)=0$ fixes the constants and the switch point.

Second vertex: Suppose $\Gamma_{11}^{1}=-1, \Gamma_{12}^{1}=1, \Gamma_{22}^{1}=-1, \Gamma_{11}^{2}=-1, \Gamma_{12}^{2}=1, \Gamma_{22}^{2}=-1$ on whole manifold. Then the auto-parallel ODE system is

$$
\ddot{x}^{1}-\left(\dot{x}^{1}-\dot{x}^{2}\right)^{2}=0, \ddot{x}^{2}-\left(\dot{x}^{1}-\dot{x}^{2}\right)^{2}=0
$$

and has a solution satisfying $x^{1}(t)=x^{2}(t)+a t+b$. This solution is

$$
x^{1}(t)=\frac{1}{2} C_{1}^{2} t^{2}+\left(C_{2}+\frac{C_{1}}{2}\right) t+C_{3}, x^{2}(t)=\frac{1}{2} C_{1}^{2} t^{2}+\left(C_{2}-\frac{C_{1}}{2}\right) t+C_{4}
$$

where $C_{1}=a, C_{3}-C_{4}=b$.

We select an auto-parallel starting from the point $\left(x\left(t_{0}\right), y\left(t_{0}\right)\right)=\left(x_{0}, y_{0}\right)$ and ending at origin $x^{1}(T)=0, x^{2}(T)=0$. Then, we find the optimal time $T=-\frac{b}{a}, a b<0$ if $b^{2}-2 C_{2} \frac{b}{a}+$ $C_{3}+C_{4}=0$. If not, we must have a switch and we must pass to a new vertex of control hyper-cube (new connection).

In case of the second vertex, we also need the dual functions $\lambda_{i}(t)$ and $\mu_{i}(t)$ : Replacing in the general formulas we find $\lambda_{i}(t)=0, i=1,2$ and $\dot{\mu}_{1}(t)=2\left(\mu_{1}(t)+\mu_{2}(t)\right)\left(-y^{1}(t)+y^{2}(t)\right)$, $\dot{\mu}_{2}(t)=2\left(\mu_{1}(t)+\mu_{2}(t)\right)\left(y^{1}(t)-y^{2}(t)\right)$. It follows $\dot{\mu}_{1}(t)+\dot{\mu}_{2}(t)=0$ or $\mu_{1}(t)+\mu_{2}(t)=c$ and 
$\mu_{1}(t)=2 c \int_{t_{0}}^{t}\left(-y^{1}(s)+y^{2}(s)\right) d s$, where $y^{i}(s)=\dot{x}^{i}(s), i=1,2$. The transversality condition $\mu_{i}\left(T^{*}\right)=0$ fixes the constants and the switch point.

To obtain the origin, we must switch each control $\gamma_{j k}^{i}(t)$ back and forth between the values \pm 1 . The optimal state path (the curve joining the starting point and the origin) is a concatenation of auto-parallel segments.

Example 4. Let us take the one-dimensional manifold $(\mathbb{R}, \Gamma(x))$, where $\Gamma(x)$ is an affine connection. The ODE of auto-parallel curves is

$$
x^{\prime \prime}(t)+\Gamma(x(t)) x^{\prime}(t)^{2}=0
$$

We consider the problem of "minimum time controlled by a connection".

If $\Gamma(x(t))=1$, then the optimal auto-parallels are $x(t)=\ln \left(c_{1} t+c_{2}\right)$. We take the initial condition $x\left(t_{0}\right)=x_{0}$, i.e., $c_{1} t_{0}+c_{2}=e^{x_{0}}$. Imposing $x(T)=0$, we find an optimal time $T=\frac{1-c_{2}}{c_{1}}$, where either $c_{1}>0, c_{2}<1$ or $c_{1}<0, c_{2}>1$. Contrary, we must have a switch and we must pass to an auto-parallel from the next category.

Let us find the dual functions $\lambda(t)$ and $\mu(t)$ in case of the first vertex: $\lambda(t)=0, \dot{\mu}(t)=$ $2 \mu y(t)$. We find $\mu(t)=c_{3} \exp \frac{2 c_{1} t}{c_{1} t+c_{2}}, t \neq \frac{-c_{2}}{c_{1}}$ (no transversality condition).

If $\Gamma(x(t))=-1$, then the optimal auto-parallels are $x(t)=-\ln \left(-c_{1} t-c_{2}\right)$. In case of the second vertex, the dual functions $\lambda(t)$ and $\mu(t)$ are the same: $\lambda(t)=0, \mu(t)=c_{3} \exp \frac{2 c_{1} t}{c_{1} t+c_{2}}, t \neq$ $\frac{-c_{2}}{c_{1}}$ (no transversality condition).

To obtain the origin, we must switch the control $\gamma(t)=\Gamma(x(t))$ back and forth between the values \pm 1 . The optimal state path (the curve joining the starting point and the origin) is a concatenation of auto-parallel segments.

\section{Another Variant of Minimum Time Problem}

In this section, the analysis is focused on single-time minimum control problems based on simple integral cost functional $J=\int_{t_{0}}^{T} d t$ and the second order ODEs system that describes the auto-parallel curves.

Objective: Find the feed-back optimal control $\gamma_{j k}^{i}(t)=\Gamma_{j k}^{i}(x(t))$ to transfer the system from a given initial condition $x\left(t_{0}\right) \in M$ to a (possibly moving) target set $S[x(T), T]$ in the shortest possible time via the functional $J=\int_{t_{0}}^{T} d t=T-t_{0}$.

State Constraints: The nonlinear second order ODEs system of auto-parallel curves is

$$
\ddot{x}^{i}(t)=-\Gamma_{j k}^{i}(x(t)) \dot{x}^{j}(t) \dot{x}^{k}(t), i, j, k=1, \ldots, n,
$$

with unknown $x(t)$. The Sturm-Liouville conditions are

$$
x\left(t_{0}\right)=x_{0}, x\left(T^{*}\right) \in S\left[x\left(T^{*}\right), T^{*}\right] .
$$

The bilocal conditions are

$$
x\left(t_{0}\right)=x_{0}, x(T)=x_{T}, \dot{x}\left(t_{0}\right)=\tilde{x}_{0}, \dot{x}(T)=\tilde{x}_{T} .
$$

The control constraints are $t \in\left[t_{0}, T\right],|\gamma(t)|=|\Gamma(x(t))| \leq 1$.

Taking $\gamma(t)=\Gamma(x(t))$ as a feedback control, we can imagine the following optimal control problem, find

$$
\min _{\gamma(t)} \int_{t_{0}}^{T} d t=T-t_{0}
$$

subject to the controlled evolution ODEs system

$$
\ddot{x}^{i}(t)=-\Gamma_{j k}^{i}(x(t)) \dot{x}^{j}(t) \dot{x}^{k}(t),|\gamma(t)|=|\Gamma(x(t))| \leq 1,
$$


with the following bilocal conditions

$$
x(0)=x_{0}, x(T)=x_{T}, \dot{x}(0)=\tilde{x}_{0}, \dot{x}(T)=\tilde{x}_{T} .
$$

Setting $X^{i}(x(t), \dot{x}(t), \gamma(t))=-\Gamma_{j k}^{i}(x(t)) \dot{x}^{j}(t) \dot{x}^{k}(t)$, we remark that the previous second order differential system can be rewritten as a first order differential system

$$
\dot{x}(t)=y(t), \dot{y}(t)=X(x(t), y(t), \gamma(t))
$$

on TM. The set of feed-back controls $\{\gamma\}$ is not a vector space. The feed-back control $\gamma(t)=\Gamma(x(t))$ enters at the degree one into the control Hamiltonian

$$
H(x(t), y(t), \gamma(t), p(t), q(t))=1+\langle p(t), y(t)\rangle+\langle q(t), X(x(t), y(t), \gamma(t))\rangle .
$$

Considering the differentiable variations $x(t, \epsilon), y(t, \epsilon)$, in fulfilling:

$$
\dot{x}(t, \epsilon)=y(t, \epsilon), \dot{y}(t, \epsilon)=X(x(t, \epsilon), y(t, \epsilon), \gamma(t)), x(t, 0)=x(t), y(t, 0)=y(t),
$$

and setting $x_{\epsilon}(t, 0)=z(t), y_{\epsilon}(t, 0)=v(t)$, we obtain the variational differential system (the summation over the repeated indices is assumed):

$$
\dot{z}(t)=v(t), \dot{v}(t)=X_{x^{j}}(x(t), y(t), \gamma(t)) z^{j}(t)+X_{y^{j}}(x(t), y(t), \gamma(t)) v^{j}(t)
$$

and the adjoint differential system

$$
\begin{gathered}
\dot{p}_{j}(t)=-\left\langle q(t), X_{x^{j}}(x(t), \dot{x}(t), \gamma(t))\right\rangle, \\
\dot{q}_{j}(t)=-p_{j}(t)-\left\langle q(t), X_{\dot{x} j}(x(t), \dot{x}(t), \gamma(t))\right\rangle .
\end{gathered}
$$

Theorem 5 (Simplified single-time minimum principle). Let $(x, \hat{\gamma})$ be an optimal pair. Then, there exist a $C^{1}$ co-state variable $p=\left(p_{i}\right)$, respectively, a $C^{2}$ co-state variable $q=\left(q_{i}\right)$, defined over $[0, T]$, such that:

$$
\begin{gathered}
\dot{x}^{j}(t)=\frac{\partial H}{\partial p_{j}}(x(t), \dot{x}(t), \hat{\gamma}(t), p(t), q(t)), \ddot{x}^{j}=\frac{\partial H}{\partial q_{j}}(x(t), \dot{x}(t), \hat{\gamma}(t), p(t), q(t)) \\
\forall t \in[0, T], j=1,2, x(0)=x_{0}, \dot{x}(0)=\tilde{x}_{0} ;
\end{gathered}
$$

the functions $p=\left(p_{i}\right), q=\left(q_{i}\right)$ satisfy the terminal problems:

$$
\begin{aligned}
& \dot{p}_{j}(t)=-H_{x^{j}}(x(t), \dot{x}(t), \hat{\gamma}(t), p(t), q(t)), p_{j}(T)=0, \\
& \dot{q}_{j}(t)=-H_{\dot{x}^{j}}(x(t), \dot{x}(t), \hat{\gamma}(t), p(t), q(t)), q_{j}(T)=0 ;
\end{aligned}
$$

the minimum condition is:

$$
H\left(x^{*}(t), y^{*}(t), \gamma(t), p^{*}(t), q^{*}(t)\right) \geq H\left(x^{*}(t), y^{*}(t), \gamma^{*}(t), p^{*}(t), q^{*}(t)\right)
$$

and the following relation

$$
\begin{gathered}
\frac{\partial H}{\partial x^{j}}(x(t), \dot{x}(t), \hat{\gamma}(t), p(t), q(t)) \\
-\frac{d}{d t}\left[\frac{\partial H}{\partial \dot{x}^{j}}(x(t), \dot{x}(t), \hat{\gamma}(t), p(t), q(t))-p_{j}(t)\right]+\frac{d^{2}}{d t^{2}}\left[-q_{j}(t)\right]=0, \forall t \in[0, T]
\end{gathered}
$$

is true.

The set of feedback controls is not a vector space. We read the following: the Hamiltonian $H(x(t), y(t), \gamma(t), p(t), q(t))$ is of degree one in the feedback control $\gamma(t)=\Gamma_{j k}^{i}(x(t))$. 
Consequently, only bang-bang control $|\gamma(t)|=\left|\Gamma_{j k}^{i}(x(t))\right| \leq 1$ is suitable for our problem. The switch coefficients are

$$
\sigma_{i}^{j k}(t)=-q_{i}(t) y^{j}(t) y^{k}(t)
$$

(decomposition similar to a tensor product).

Finding the Optimal Control (Bang-Bang control): the minimum condition of Hamiltonian is equivalent to a maximum condition

$$
q_{i}^{*}(t) \gamma_{j k}^{* i}(t) y^{* j}(t) y^{* k}(t) \geq q_{i}^{*}(t) \gamma_{j k}^{i}(t) y^{* j}(t) y^{* k}(t), \forall \text { admissible } \gamma(t) .
$$

On the other hand,

$$
\max _{\left|\gamma_{j k}^{i}(t)\right| \leq 1} q_{i}^{*}(t) \gamma_{j k}^{i}(t) y^{* j}(t) y^{* k}(t)=\left|q_{i}^{*}(t) y^{* j}(t) y^{* k}(t)\right|
$$

and the optimal connections are those with particular constant feedback

$$
\gamma_{j k}^{* i}(t)=\operatorname{sign}\left(-q_{i}^{*}(t) y^{* j}(t) y^{* k}(t)\right) .
$$

Connections with particular constant coefficients are examples. Control is always at its extremum value.

\section{Stochastic Connectivity}

\subsection{Pfaff Form of Auto-Parallel ODEs}

The previous nonlinear control system (2) can be written as a Pfaff system

$$
d x^{i}(t)=y^{i}(t) d t, d y^{i}(t)=-\Gamma_{j k}^{i}(x(t)) y^{j}(t) y^{k}(t) d t
$$

on $T M \times \mathbb{R}$. We introduce an $n$-dimensional vector field $\mathbf{0}=(0,0, \ldots, 0)$ and denote $\xi^{i}=\Gamma_{j k}^{i}(x) y^{j} y^{k}$. On $\widehat{T M}=T M \backslash\{0\}$, it appears $2 n$ vector fields normal to auto-parallel distribution

$$
\begin{gathered}
\left(-1,0, \ldots, 0 ; \mathbf{0} ; y^{1}\right),\left(0,-1, \ldots, 0 ; \mathbf{0} ; y^{2}\right), \ldots,\left(0,0, \ldots,-1 ; \mathbf{0} ; y^{n}\right), \\
\left(\mathbf{0} ;-1,0, \ldots, 0 ;-\xi^{1}\right),\left(\mathbf{0} ; 0,-1, \ldots, 0 ;-\xi^{2}\right), \ldots,\left(\mathbf{0} ; 0, \ldots,-1 ;-\xi^{n}\right)
\end{gathered}
$$

and one vector field tangent to auto-parallel distribution (orthogonal to the previous vector fields)

$$
\left(y^{1}, \ldots, y^{n} ;-\xi^{1}, \ldots,-\xi^{n} ; 1\right) .
$$

\subsection{Stochastic Perturbations}

We study the impact of stochastic perturbations to previous deterministic Pfaff dynamical system of auto-parallels and explore how stochastic noise can be used to find connectivity properties generated by the underlying deterministic auto-parallel dynamics. The stochastic perturbation of ODEs system (2) is defined by

$$
\begin{gathered}
d x^{i}(t)=y^{i}(t) d t+\sigma_{a}^{i}(t, x(t), y(t)) d W^{a}(t), \\
d y^{i}(t)=-\Gamma_{j k}^{i}(x(t)) y^{j}(t) y^{k}(t) d t+\eta_{a}^{i}(t, x(t), y(t)) d W^{a}(t), i, j, k=1, \ldots, n,
\end{gathered}
$$

where $W^{a}(t), t \geq t_{0}, a=1, \ldots, d$, are independent Wiener processes, $y^{i}(t),-\Gamma_{j k}^{i}(x(t)) y^{j}(t)$ $y^{k}(t)$ are drift coefficients and $\sigma_{a}^{i}(t, x(t), y(t)), \eta_{a}^{i}(t, x(t), y(t))$ are diffusion coefficients.

For general theory regarding stochastic differential equations (SDE), see [20]. 
Definition 4. A strong solution of this stochastic differential system with initial condition $\left(x\left(t_{0}\right)\right.$, $\left.y\left(t_{0}\right)\right) \in T M$ is an adapted continuous process $c(t)=(x(t), y(t))$ such that for all $t \geq 0$, it satisfies

$$
\begin{gathered}
x^{i}(t)-x^{i}\left(t_{0}\right)=\int_{t_{0}}^{t} y^{i}(s) d s+\int_{t_{0}}^{t} \sigma_{a}^{i}(s, x(s), y(s)) d W^{a}(s) \\
y^{i}(t)-y^{i}\left(t_{0}\right)=-\int_{t_{0}}^{t} \Gamma_{j k}^{i}(x(s)) y^{j}(s) y^{k}(s) d s+\int_{t_{0}}^{t} \eta_{a}^{i}(s, x(s), y(s)) d W^{a}(s), \text { a.s. }
\end{gathered}
$$

At first sight this definition seems to have little content except to provide a more-or-less obvious interpretation of the SDE. However, there are a number of subtle points involved: first, the existence of the integrals requires some degree of regularity on $c(t)=(x(t), y(t))$ and on the functions $y, \sigma$, and $\eta$; in particular, it must be the case that for all $t \geq t_{0}$ with probability one, we must have

$$
\int_{t_{0}}^{t}\|y(s)\| d s<\infty, \int_{t_{0}}^{t}\|\sigma(s)\|^{2} d s<\infty .
$$

Second, the solution is required to exist for all $t<\infty$ with probability one. In fact, there are interesting cases for which solutions can be constructed up to a finite and possibly random time $T<\infty$, but not beyond; this often happens because the solution $(x(t), y(t))$ explodes (that is, runs off to $\pm \infty$ ) in finite time.

Definition 5. A weak solution of the SDE with i.c. $\left(x\left(t_{0}\right), y\left(t_{0}\right)\right)$ is a continuous stochastic process $c(t)=(x(t), y(t))$ defined on some probability space $(\Omega, \mathcal{F}, P)$, such that for some Wiener processes $W^{a}(t)$ and some admissible filtration $\mathcal{F}$ the process $c(t)=(x(t), y(t))$ is adapted and satisfies the associated stochastic integral system.

The basic result, due to Itô, is that for uniformly Lipschitz functions $\Gamma, \sigma$ the SDE has strong solutions and that for each initial value $c\left(t_{0}\right)=\left(x\left(t_{0}\right), y\left(t_{0}\right)\right)$ the solution is unique.

We use the modulus $S p\left\{d W^{a}\right\}$ over the ring of continuous functions. On $\widetilde{T M} \times$ $S p\left\{d W^{a}\right\}$, it appears $2 n$ "vector fields" normal to stochastic auto-parallel distribution

$$
\begin{aligned}
& \left(-1,0, \ldots, 0 ; \mathbf{0} ; y^{1} ; \sigma_{1}^{1}, \ldots, \sigma_{d}^{1}\right), \ldots,\left(0,0, \ldots,-1 ; \mathbf{0} ; y^{n} ; \sigma_{1}^{n}, \ldots, \sigma_{d}^{n}\right), \\
& \left(\mathbf{0} ;-1,0, \ldots, 0 ;-\xi^{1} ; \eta_{1}^{1}, \ldots, \eta_{d}^{1}\right), \ldots,\left(\mathbf{0} ; 0, \ldots,-1 ;-\xi^{n} ; \eta_{1}^{n}, \ldots, \eta_{d}^{n}\right)
\end{aligned}
$$

and $1+d$ "vector fields" tangent to stochastic auto-parallel distribution (orthogonal to the previous vectors), between which the vector field

$$
\left(y^{1}, \ldots, y^{n} ;-\xi^{1}, \ldots,-\xi^{n} ; 1 ;(0, \ldots, 0)\right)
$$

and other $d$ "vector fields".

\subsection{Simplified Stochastic Minimum Principle}

The problem is to find:

$$
\min _{\gamma_{j k}^{i}(t)} \int_{t_{0}}^{T} d t=T-t_{0}
$$

subject to the controlled evolution stochastic ODEs system

$$
\begin{gathered}
d x^{i}(t)=y^{i}(t) d t+\sigma_{a}^{i}(t, x(t), y(t)) d W^{a}(t) \\
d y^{i}(t)=-\Gamma_{j k}^{i}(x(t)) y^{j}(t) y^{k}(t) d t+\eta_{a}^{i}(t, x(t), y(t)) d W^{a}(t), \\
\left|\gamma_{j k}^{i}(t)\right|=\left|\Gamma_{j k}^{i}(x(t))\right| \leq 1,
\end{gathered}
$$


with the following bilocal conditions

$$
x\left(t_{0}\right)=x_{0}, x(T)=x_{T}, y\left(t_{0}\right)=\tilde{y}_{0}, y(T)=\tilde{y}_{T},
$$

can be solved via the next stochastic minimum principle.

Let $t \in\left[t_{0}, T\right]$. Let $x(t)$ be the stochastic or diffusion process, $u(t)$ be the random control variable, $\mu^{i}(t, x(t), u(t))$ be a drift vector field, $\sigma=\left(\sigma_{a}^{i}(t, x(t), u(t))\right)$ be a diffusion matrix function, and $d W^{a}(t)$ be a finite dimensional Wiener process. A standard stochastic optimal control problem is formulated in the following manner [21,22]:

$$
\begin{gathered}
I(u(\cdot))=E\left\{\int_{t_{0}}^{T} L(t, x(t), u(t)) d t+K(T, x(T))\right\} \\
\min _{u} I(u(\cdot)), \text { s.t. } \\
d x^{i}(t)=\mu^{i}(t, x(t), u(t)) d t+\sigma_{a}^{i}(t, x(t), u(t)) d W^{a}(t),
\end{gathered}
$$

where $x\left(t_{0}\right)=x_{0} \in \mathbb{R}^{n}, i=1, \ldots, n ; a=1, \ldots, d$. The stochastic process $x(t)$ is usually a Markov process.

We proved a version of the minimum principle in the sense of Pontryagin for the optimal control of a stochastic differential equation driven by a finite dimensional Wiener process $[21,22]$. Our version of stochastic minimum principle is based on the following ideas: (i) the dual variable is of the form $p(t)$; (ii) the stochastic first integral is a good ingredient for defining the dual stochastic equations; (iii) a geometrical formalism, using the integrand 1-form, in order to underline the idea of possible changing the single-time problems theory into multitime problems theory (objective functionals given by curvilinear integrals or multiple integrals (integrals from $q$-forms), constrained by multitime Itô evolution equations). The adjective "simplified" underlines the hypothesis that the optimal control is in the "interior" of the set of controls and that the variational calculus techniques can be applied.

In our context, we use a control Hamiltonian stochastic 1-form

$$
\mathcal{H}(t, x, u, p)=\left[L(t, x, u)+p_{i}\left(\mu^{i}(t, x, u)-\sigma_{a x^{j}}^{i}(t, x, u) \sigma_{b}^{j}(t, x, u) \delta^{a b}\right)\right] d t
$$

and its pullback (see the Einstein summation convention).

Theorem 6 (Simplified stochastic minimum principle). Suppose that the previous optimal problem has an interior optimal solution $u^{*}(t)$, which determines the stochastic optimal evolution $x(t)$. Let $\mathcal{H}$ be the Hamiltonian stochastic 1 -form. Then there exists an adapted process $p(t)=$ $\left(p_{i}(t)\right), t \in\left[t_{0}, T\right]$ (adjoint stochastic process) satisfying the following

(i) The initial stochastic differential system

$$
\begin{gathered}
d x^{i}(t)=\frac{\partial \mathcal{H}}{\partial p_{i}}\left(t, x(t), u^{*}(t), p(t)\right)+\sigma_{a x j}^{i}\left(t, x(t), u^{*}(t)\right) \sigma_{b}^{j}\left(t, x(t), u^{*}(t)\right) \delta^{a b} d t \\
+\sigma_{a}^{i}\left(t, x(t), u^{*}(t)\right) d W^{a}(t) ;
\end{gathered}
$$

(ii) The adjoint linear stochastic differential system

$$
\begin{gathered}
d p_{i}(t)=-\mathcal{H}_{x^{i}}\left(t, x(t), u^{*}(t), p(t)\right)-p_{j}(t) \sigma_{a x^{i}}^{j}\left(t, x(t), u^{*}(t)\right) d W^{a}(t), \\
p_{i}(T)=E\left(K_{x^{i}}(T, x(T)) ;\right.
\end{gathered}
$$

(iii) The minimum point condition

$$
\min _{u} \mathcal{H}(t, x(t), u, p(t))=\mathcal{H}\left(t, x(t), u^{*}, p(t)\right)
$$


or the critical point condition

$$
\mathcal{H}_{u^{c}}\left(t, x(t), u^{*}, p(t)\right)=0, c=1, \ldots, k
$$

\subsection{Striking Time}

To simplify, we consider that the amplitude of errors are constant matrices, i.e., $\sigma_{a}^{i}(t, x(t), y(t))=\sigma_{a}^{i}, \eta_{a}^{i}(t, x(t), y(t))=\eta_{a}^{i}$ and that the SDE are

$$
d x^{i}(t)=y^{i}(t) d t+\sigma_{a}^{i} d W^{a}(t), d y^{i}(t)=-\Gamma_{j k}^{i}(x(t)) y^{j}(t) y^{k}(t) d t+\eta_{a}^{i} d W^{a}(t) .
$$

Definition 6. A stochastic process $c(t)=(x(t), y(t))$, which satisfies the SDE system, where $\gamma_{j k}^{i}(t)=\Gamma_{j k}^{i}(x(t))$ is a feedback bounded control and is called an admissible stochastic process.

Theorem 7. Let us consider a compact Riemannian manifold $(M, g)$, with positive sectional curvature. The Sasaki tangent bundle is $(T M, G=g \oplus g)$. Let $P=\left(x_{P}, y_{P}\right), Q=\left(x_{Q}, y_{Q}\right)$ be two points on the manifold TM. Denote $D(Q, r)$ the Riemannian disk of radius $r$, centered at $Q$ in $(T M, G)$. Then, for any $\epsilon, r>0$, there exists a striking time $t<\infty$ and an admissible stochastic process $c(t)$, satisfying the boundary conditions

$$
\left(x\left(t_{0}\right), y\left(t_{0}\right)\right)=\left(x_{P}, y_{P}\right),(E[x(t)], E[y(t)])=\left(x_{Q}, y_{Q}\right),
$$

such that

$$
\mathbb{P}(c(t) \in D(Q, r)) \geq 1-\epsilon .
$$

Proof. The manifold $(M, g)$ is geodesically complete.

We start with the non-negative random variable $d^{2}(Q, c(t))$. The Markov inequality shows that

$$
\mathbb{P}\left(d^{2}(Q, c(t)) \geq r^{2}\right) \leq \frac{1}{r^{2}} \mathbb{E}\left[d^{2}(Q, c(t))\right]
$$

or, equivalently,

$$
\mathbb{P}\left(d^{2}(Q, c(t)) \leq r^{2}\right) \geq 1-\frac{1}{r^{2}} \mathbb{E}\left[d^{2}(Q, c(t))\right] .
$$

The integral variant of the previous SDE system is

$$
\begin{gathered}
x^{i}(t)=\int_{t_{0}}^{t} y^{i}(s) d s+\sigma_{a}^{i} W^{a}(t)+x_{P}^{i}, \\
y^{i}(t)=-\int_{t_{0}}^{t} \Gamma_{j k}^{i}(x(s)) y^{j}(s) y^{k}(s) d s+\eta_{a}^{i} W^{a}(t)+y_{P}^{i} .
\end{gathered}
$$

It is well-known that

$$
\mathbb{E}\left[W^{a}(t)\right]=0, \mathbb{E}\left[\left(W^{a}(t)\right)^{2}\right]=t, \mathbb{E}\left[W^{a}(t) W^{b}(t)\right]=0, a \neq b .
$$

If two random variables $X, Y: \Omega \rightarrow \mathbb{R}$ are independent, then $\mathbb{E}[X Y]=\mathbb{E}[X] \mathbb{E}[Y]$, provided that $\mathbb{E}[|X|]<\infty$ and $\mathbb{E}[|Y|]<\infty$. Moreover, the boundary conditions

$$
\begin{gathered}
\mathbb{E}\left[x^{i}(t)\right]=x_{Q}^{i} \Rightarrow \int_{t_{0}}^{t} \mathbb{E}\left[y^{i}(s)\right] d s+x_{P}^{i}=x_{Q}^{i} \\
\mathbb{E}\left[y^{i}(t)\right]=y_{Q}^{i} \Rightarrow-\int_{t_{0}}^{t} \mathbb{E}\left[\Gamma_{j k}^{i}(x(s)) y^{j}(s) y^{k}(s)\right] d s+y_{P}^{i}=y_{Q}^{i} .
\end{gathered}
$$

The positivity of the curvature of a Riemannian manifold $(M, g)$ implies the fact that the distance on the manifold is at most the distance in the tangent space, [23], i.e., $d_{g}(Q, c(t)) \leq d_{\mathbb{R}^{n}}(Q, c(t))$. Moreover, to simplify, we accept $t_{0}=0$. Based on the indepen- 
dence of $W^{a}(t)$, we find the following sequence of relations (starting with Pythagorean theorem)

$$
\begin{aligned}
& \mathbb{E}\left[d^{2}(Q, c(t))\right]=\mathbb{E}\left[d^{2}\left(x_{Q}, x(t)\right)\right]+\mathbb{E}\left[d^{2}\left(y_{Q}, y(t)\right)\right] \\
& \leq \mathbb{E}\left[\delta_{i j}\left(x^{i}(t)-x_{Q}^{i}\right)\left(x^{j}(t)-x_{Q}^{j}\right)\right]+\mathbb{E}\left[\delta_{i j}\left(y^{i}(t)-y_{Q}^{i}\right)\left(y^{j}(t)-y_{Q}^{j}\right)\right] \\
& =\mathbb{E}\left[\delta_{i j}\left(\int_{0}^{t} y^{i}(s) d s+\sigma_{a}^{i} W^{a}(t)+x_{P}^{i}-x_{Q}^{i}\right)\right. \\
& \left.\times\left(\int_{0}^{t} y^{j}(s) d s+\sigma_{b}^{j} W^{b}(t)+x_{P}^{j}-x_{Q}^{j}\right)\right] \\
& +\mathbb{E}\left[\delta_{i j}\left(-\int_{0}^{t} \Gamma_{k m}^{i}(x(s)) y^{k}(s) y^{m}(s) d s+\eta_{a}^{i} W^{a}(t)+y_{P}^{i}-y_{Q}^{i}\right)\right. \\
& \left.\times\left(-\int_{0}^{t} \Gamma_{p q}^{j}(x(s)) y^{p}(s) y^{q}(s) d s+\eta_{b}^{j} W^{b}(t)+y_{P}^{j}-y_{Q}^{j}\right)\right] \\
& =\mathbb{E}\left[\delta_{i j} \int_{0}^{t} y^{i}(s) d s \int_{t_{0}}^{t} y^{j}(s) d s+\delta_{i j} \sigma_{a}^{i} W^{a}(t) \sigma_{b}^{j} W^{b}(t)+\delta_{i j} x_{P}^{i} x_{P}^{j}+\delta_{i j} x_{Q}^{i} x_{Q}^{j}\right. \\
& +2 \delta_{i j}\left(\int_{0}^{t} y^{i}(s) d s\right) \sigma_{a}^{j} W^{a}(t)+2 \delta_{i j}\left(\int_{0}^{t} y^{i}(s) d s\right) x_{P}^{j} \\
& \left.-2 \delta_{i j}\left(\int_{0}^{t} y^{i}(s) d s\right) x_{Q}^{j}+2 \delta_{i j} \sigma_{a}^{i} W^{a}(t) x_{P}^{j}-2 \delta_{i j} \sigma_{a}^{i} W^{a}(t) x_{Q}^{j}-2 \delta_{i j} x_{P}^{i} x_{Q}^{j}\right] \\
& +\mathbb{E}\left[\delta_{i j} \int_{0}^{t} \Gamma_{k m}^{i}(x(s)) y^{k}(s) y^{m}(s) d s \int_{0}^{t} \Gamma_{p q}^{j}(x(s)) y^{p}(s) y^{q}(s) d s\right. \\
& +\delta_{i j} \eta_{a}^{i} W^{a}(t) \eta_{b}^{j} W^{b}(t)+\delta_{i j} y_{P}^{i} y_{P}^{j}+\delta_{i j} y_{Q}^{i} y_{Q}^{j} \\
& -2 \delta_{i j}\left(\int_{0}^{t} \Gamma_{k m}^{i}(x(s)) y^{k}(s) y^{m}(s) d s\right) \eta_{b}^{j} W^{b}(t) \\
& -2 \delta_{i j}\left(\int_{0}^{t} \Gamma_{k m}^{i}(x(s)) y^{k}(s) y^{m}(s) d s\right) y_{P}^{j} \\
& +2 \delta_{i j}\left(\int_{0}^{t} \Gamma_{k m}^{i}(x(s)) y^{k}(s) y^{m}(s) d s\right) y_{Q}^{j}+2 \delta_{i j} \sigma_{a}^{i} W^{a}(t) y_{P}^{j} \\
& \left.-2 \delta_{i j} \sigma_{a}^{i} W^{a}(t) y_{Q}^{j}-2 \delta_{i j} y_{P}^{i} y_{Q}^{j}\right] .
\end{aligned}
$$

This last expression is reduced to

$$
\begin{gathered}
\mathbb{E}\left[\delta_{i j} \int_{0}^{t} y^{i}(s) d s \int_{0}^{t} y^{j}(s) d s\right]+\delta_{i j} t \sum_{a} \sigma_{a}^{i} \sigma_{a}^{j}-\delta_{i j}\left(x_{P}^{i}-x_{Q}^{i}\right)\left(x_{P}^{j}-x_{Q}^{j}\right) \\
+\mathbb{E}\left[\delta_{i j} \int_{0}^{t} \Gamma_{k m}^{i}(x(s)) y^{k}(s) y^{m}(s) d s \int_{0}^{t} \Gamma_{p q}^{j}(x(s)) y^{p}(s) y^{q}(s) d s\right] \\
+\delta_{i j} t \sum_{a} \eta_{a}^{i} \eta_{a}^{j}-\delta_{i j}\left(y_{P}^{i}-y_{Q}^{i}\right)\left(y_{P}^{j}-y_{Q}^{j}\right) .
\end{gathered}
$$

Since $M$ is compact, we can suppose

$$
\left|\Gamma_{k m}^{i}(x(s)) y^{k}(s) y^{m}(s)\right| \leq a \delta_{k m} y^{k}(s) y^{m}(s)=a\|y(s)\|_{\delta}^{2} .
$$

Then, the Cauchy-Schwarz inequalities are

$$
\delta_{i j} \int_{0}^{t} y^{i}(s) d s \int_{0}^{t} y^{j}(s) d s \leq \int_{0}^{t} \delta_{i j} y^{i}(s) y^{j}(s) d s \int_{0}^{t} d s,
$$




$$
\begin{aligned}
& \delta_{i j} \int_{0}^{t} \Gamma_{k m}^{i}(x(s)) y^{k}(s) y^{m}(s) d s \int_{0}^{t} \Gamma_{p q}^{j}(x(s)) y^{p}(s) y^{q}(s) d s \\
\leq & \int_{0}^{t} \delta_{i j} \Gamma_{k m}^{i}(x(s)) y^{k}(s) y^{m}(s) \Gamma_{p q}^{j}(x(s)) y^{p}(s) y^{q}(s) d s \int_{0}^{t} d s
\end{aligned}
$$

and imply

$$
\begin{gathered}
\mathbb{E}\left[\delta_{i j} \int_{0}^{t} y^{i}(s) d s \int_{0}^{t} y^{j}(s) d s\right] \leq t \int_{0}^{t} \delta_{i j} \mathbb{E}\left[y^{i}(s)\right] \mathbb{E}\left[y^{j}(s)\right] d s \leq\left\|y_{Q}\right\|^{2} t^{2}, \\
\mathbb{E}\left[\delta_{i j} \int_{0}^{t} \Gamma_{k m}^{i}(x(s)) y^{k}(s) y^{m}(s) d s \int_{0}^{t} \Gamma_{p q}^{j}(x(s)) y^{p}(s) y^{q}(s) d s\right] \\
\leq t \int_{0}^{t} \delta_{i j} \mathbb{E}\left[\Gamma_{k m}^{i}(x(s)) y^{k}(s) y^{m}(s)\right] \mathbb{E}\left[\Gamma_{p q}^{j}(x(s)) y^{p}(s) y^{q}(s)\right] d s \leq k\left\|y_{Q}\right\|^{4} t^{2} .
\end{gathered}
$$

Consequently, we have

$$
\mathbb{E}\left[d^{2}(Q, c(t))\right] \leq\left(k_{1}^{2}+k_{2}^{2}\right) t^{2}+\|\sigma\|^{2} t-\left\|x_{P}-x_{Q}\right\|^{2}-\left\|y_{P}-y_{Q}\right\|^{2} .
$$

For any $\epsilon, r>0$, the following equation

$$
\left(k_{1}^{2}+k_{2}^{2}\right) t^{2}+\|\sigma\|^{2} t-\left\|x_{P}-x_{Q}\right\|^{2}-\left\|y_{P}-y_{Q}\right\|^{2}-\epsilon r^{2}=0
$$

has a strictly positive solution

$$
t=\frac{-\|\sigma\|^{2}+\sqrt{\|\sigma\|^{4}+4\left(k_{1}^{2}+k_{2}^{2}\right) \Lambda^{2}}}{2\left(k_{1}^{2}+k_{2}^{2}\right)},
$$

where $\Lambda^{2}=\left\|x_{P}-x_{Q}\right\|^{2}+\left\|y_{P}-y_{Q}\right\|^{2}+\epsilon r^{2}$.

The root $t$ is called striking time. For this $t$ we can write

$$
\mathbb{E}\left[d^{2}(Q, c(t))\right] \leq \epsilon r^{2} \Leftrightarrow 1-\frac{1}{r^{2}} \mathbb{E}\left[d^{2}(Q, c(t))\right] \geq 1-\epsilon,
$$

i.e.,

$$
\mathbb{P}(c(t) \in D(Q, r)) \geq 1-\epsilon .
$$

\subsection{Striking Time Estimation}

Changing notations in mind, we use the NLPSolve command of Maple to find a striking time estimation (local minimum). They are described as follows

$>$ with(Optimization);

$>$ NLPSolve $\left(\left(-\sigma^{2}+\sqrt{\sigma^{4}+4 * \alpha^{2} *\left(\epsilon * r^{2}+\beta^{2}\right)}\right) /\left(2 * \alpha^{2}\right)\right.$,

$\epsilon=0.01 . .0 .04, r=1 . .7, \alpha=5 . .7, \beta=1 . .6)$;

$\left[2.38418579101562546 * 10^{-9},[\alpha=5 ., \beta=1 ., \epsilon=0.01\right.$,

$r=1.70262548508052, \sigma=18822.5365605649]]$

$t_{\min }=2.38418579101562546 \times 10^{-9}$.

Striking time estimation is required for papers in discrete mathematics.

\section{Conclusions}

In this paper, we proposed a new point of view at intersection of Differential Geometry and Optimal Control, namely controllability and observability via auto-parallel curves. Although surprising for both scientific fields, this problem had to be raised because the applications on affine manifolds are plausible and have a high scientific impact. Customizing the controlled ODEs system is a well-known technique and this point of view is well-related to applications (see also [11,12,19]). 
Bang-Bang control is a type of control system that mechanically or electronically turns something on or off when a desired target has been reached. Bang-bang controls frequently arise in minimum-time problems, if the Hamiltonian is of degree one in the control variable.

Our paper underlines that several techniques (coming from Differential Geometry, Optimal Control, and Stochastic Dynamical Systems) are necessary to be put together in order to create original results.

The examples from this paper allows us to appreciate the point of view of the Romanian geometer G. Vranceanu regarding the existence of constant connections and their role in differential geometry.

The terminology in optimal control was intertwined with the terminology in differential geometry, which motivates the title of the paper. This title is understood only in context. Broken context is intriguing.

In a forthcoming paper, the proposed idea will be develop further since the controllability and observability, both as dual aspects of the same problem, are very important in applied sciences.

Author Contributions: Conceptualization, C.U. and I.T.; writing—original draft preparation, C.U.; writing-review and editing, C.U. and I.T. Both authors have read and agreed to the published version of the manuscript.

Funding: This research received no external funding.

Informed Consent Statement: Not applicable.

Data Availability Statement: The data used to support the findings of this paper are included within the article and are available from the corresponding author upon request.

Acknowledgments: We thank MDPI for their interest in our paper and the reviewers for pertinent suggestions. Best wishes go to Miss Associate Oana-Maria Pastae, "Constantin Brancusi" University of Tg-Jiu, for the English improvement of the manuscript.

Conflicts of Interest: The authors declare no conflict of interest.

\section{References}

1. Nomizu, K. On Completeness in Affine Differential Geometry. Geom. Dedicata 1986, 20, 43-49. [CrossRef]

2. Pesch, H.J.; Bechmann, S.; Wurst, J.-E. Bang-Bang and Singular Controls in Optimal Control Problems with Partial Differential Equations. In Proceedings of the 2012 IEEE 51st IEEE Conference on Decision and Control, Maui, HI, USA, 10-13 December 2012. [CrossRef]

3. Evans, L.C. An Introduction to Mathematical Optimal Control Theory; Internet; University of California: Berkeley, CA, USA, 2021.

4. Kim, J.-H.R.; Maurer, H.; Astrov, Y.A.; Bode, M. High speed switch-on of a semiconductor gas discharge image converter using optimal control methods. J. Comput. Phys. 2001, 170, 395-414. [CrossRef]

5. Udriste, C.; Dinu, S.; Tevy, I. Multitime optimal control for linear PDEs with curvilinear cost functional. Balkan J. Geom. Appl. 2013, 18, 87-100.

6. Olsder, G.J. An open- and closed-loop bang-bang control in nonzero-sum differential games. SIAM J. Control Optim. 2001, 40, 1087-1106. [CrossRef]

7. Dadebo, S.A.; McAuley, K.B.; McLellan, P.J. On the computation of optimal singular and bang-bang controls. Optim. Control Appl. Methods 1998, 19, 287-297. [CrossRef]

8. Ledzewicz, U.; Schättler, H. Optimal bang-bang controls for a two compartment model in cancer chemotherapy. J. Optim. Theory Appl. 2002, 114, 609-637. [CrossRef]

9. Kaya, C.Y. Time-Optimal Switching Control for the U.S. Cocaine Epidemic; Socio-Economic Planning Sciences; Elsevier: 2004; Volume 38, pp. 57-72. Available online: https:/ /www.sciencedirect.com/science/article/abs/pii/S0038012103000284 (accessed on 4 April 2021).

10. Chui, C.K.; Chen, G. Minimum-Time Optimal Control Problems; Chapter in Linear Systems and Optimal Control; Springer: Berlin/Heidelberg, Germany, 1989; pp. 94-105.

11. Bloch, A.M.; Gupta, R.; Kolmanovsky, I.V. Neighboring extremal optimal control for mechanical systems on Riemannian manifolds. J. Geom. Mech. Inst. Math. Sci. 2016, 8, 257-272. [CrossRef]

12. Sun, Y. Linear controllability versus global controllability. IEEE Trans. Autom. Control 2009, 54, 1693-1697.

13. Lewis, A.D. Affine Connection Control Systems; Copyright and IFAC, Lagrangian and Hamiltonian Methods for Nonlinear Control: Princeton, NJ, USA, 2000. 
14. Lewis, A.D. The category of affine connection control systems. In Proceedings of the 39th IEEE Conference on Decision and Control, Sydney, Australia, 12-15 December 2000; pp. 5119-5124. [CrossRef]

15. Hirica, I.; Udriste, C.; Pripoae, G.; Tevy, I. Least squares approximation of flatness on Riemannian manifolds. Mathematics 2020, 8 , 1757. [CrossRef]

16. Hirica, I.; Udriste, C.; Pripoae, G.; Tevy, I. Riccati PDEs that imply curvature-flatness. Mathematics 2021, 9, 537. [CrossRef]

17. Kobayashi, S.; Nomizu, K. Foundations of Differential Geometry; John Wiley and Sons: New York, NY, USA, 1963; Volume I.

18. Huijberts, H.; Nijmeijer, H. Controllability and Observability of Nonlinear Systems; Control Systems, Robotics, and Automation; EOLSS Publishers: Oxford, UK, 2018; Volume XII.

19. Pardalos, P.; Yatsenko, V. Optimization and Control of Bilinear Systems: Theory, Algorithms, and Applications; Springer: New York, NY, USA, 2008.

20. Calin, O. An Informal Introduction to Stochastic Calculus with Applications; World Scientific Publishing: Singapore, 2015; ISBN 978981-4678-93-3.

21. Udriste, C. Comparing variants of single-time stochastic maximum principle. In Proceedings of the 4th International Conference on Applied and Computational Mathematics (ICACM '15), Seoul, Korea, 5-7 September 2015.

22. Udriste, C.; Damian, V. Simplified single-time stochastic maximum principle. Balkan J. Geom. Appl. 2011, 16, 155-173.

23. Udriste, C. Sufficient decrease principle on Riemannian manifolds. Balkan J. Geom. Appl. 1996, 1, 111-123. 